\title{
Routine post-weaning handling of rats prevents isolation rearing-induced deficit in prepulse inhibition
}

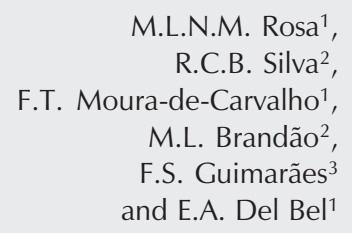

M.L.N.M. Rosa ${ }^{1}$,

R.C.B. Silva²,

F.T. Moura-de-Carvalho ${ }^{1}$,

M.L. Brandão ${ }^{2}$,

F.S. Guimarães ${ }^{3}$

and E.A. Del Bel ${ }^{1}$

\author{
'Departamento de Morfologia, Estomatologia e Fisiologia, \\ Faculdade de Odontologia de Ribeirão Preto, Universidade de São Paulo, \\ Ribeirão Preto, SP, Brasil \\ ${ }^{2}$ Departamento de Psicologia, Faculdade de Filosofia, \\ Ciências e Letras de Ribeirão Preto, Universidade de São Paulo, \\ Ribeirão Preto, SP, Brasil \\ ${ }^{3}$ Departamento de Farmacologia, Faculdade de Medicina de Ribeirão Preto, \\ Universidade de São Paulo, Ribeirão Preto, SP, Brasil
}

\section{Correspondence \\ M.L.N.M. Rosa \\ Departamento de Morfologia, \\ Estomatologia e Fisiologia \\ FORP, USP \\ Av. do Café, $\mathrm{s} / \mathrm{n}$ \\ 14040-904 Ribeirão Preto, SP \\ Brasil \\ Fax: +55-16-633-0999 \\ E-mail: mlrosa.fameca@gmail.com}

Research supported by FAPESP (No. 98/10639-7) and CNPq. M.L.N.M. Rosa is the recipient of a FAPESP postdoctoral fellow (No. 00/12108-0).

Received September 23, 2004 Accepted July 27, 2005

\begin{abstract}
Rats reared under isolation conditions from weaning present a number of behavioral changes compared to animals reared under social conditions (group housing). These changes include deficits in prepulse inhibition (PPI) of the startle reflex to a loud sound. PPI refers to the reduction of the magnitude of the startle reflex when a relatively weak stimulus (the prepulse) precedes by an appropriate time interval the intense startle-elicing stimulus (the pulse). PPI is useful for studying sensorimotor integration. The present study evaluated the effect of handling on the impairment of PPI induced by isolation-rearing. Male Wistar rats ( $\mathrm{N}=11-15 /$ group) were housed in groups (5 per cage and handled three times a week) or isolated (housed individually) since weaning (21 days) for 10 weeks when they reach approximately 150 g. The isolated rats were divided into "minimally handled" animals (handled once a week for cleaning purposes only) or "handled" animals (handled three times a week). This handling consisted of grasping the rat by the tail and moving it to a clean cage (approximately $5 \mathrm{~s}$ ). A statistically significant reduction $(52 \%)$ in the PPI test was found only in the isolated group with minimal handling while no difference was seen between grouped animals and isolated handled animals. These results indicate that isolation rearing causes disruption in the PPI at adult age, which serves as an index of attention deficit. This change in the sensory processing of information induced by postweaning isolation can be prevented by handling during the development of the animal.
\end{abstract}

Key words

- Stress

- Rat

- Schizophrenia

- Social isolation

- Prepulse inhibition

- Locomotor activity

.......
Animal models are important tools for the advancement of medicine and are of increasing importance in psychiatry (1). Social isolation is considered to be a chronic source of stress and its effects have been related to alcoholism, pathologic depression, anxiety, and schizophrenia $(1,2)$. Rats reared under isolation conditions from the end of weaning present a variety of changes in the central nervous system, probably reflected 
by several physiological alterations such as increased plasma corticosterone and brain corticotrophin-releasing hormone concentrations (1). Furthermore, isolated animals present a number of behavioral changes. They are hyperactive in a novel environment, display deficits in prepulse inhibition (PPI) of the startle response and auditory gating and show enhanced mesolimbic dopamine (DA) function $(3,4)$.

PPI refers to the reduction in the startle response produced when a low-intensity nonstartling stimulus (the prepulse) is presented shortly before the startle stimulus (the pulse). The PPI test has attracted considerable interest in recent years since it may measure attentional impairments associated with psychiatric disorders. Deficient PPI has been reported in schizophrenia, obsessive compulsive disorder, Huntington's and Parkinson's diseases, and Tourette disorder, among others. These disorders are characterized symptomatically by a loss of gating in sensory, motor, or cognitive domains, and by abnormalities in the cortico-striato-pallido-pontine circuitry that modulate PPI (5).

Evidence shows that PPI is regulated by DA and/or serotonergic (5-HT) circuitry (5). DA overactivity in limbic areas has been related to both clinical symptoms of schizophrenia and PPI deficit. In addition, the PPI deficit produced by isolation rearing or administration of direct (apomorphine) or indirect (d-amphetamine) DA agonists is reversed by treatment with typical or atypical antipsychotics $(5,6)$.

Several factors can influence PPI, probably accounting for the different results sometimes found between laboratories. These include rat strain (7), development stage (8), duration of isolation (9), which should be continuous (10), cage type (11) and test sequence (12). Care methods could also potentially prevent some of the behavioral changes induced by social isolation $(13,14)$ and it has recently been suggested that postweaning handling can attenuate deficits in
PPI induced by isolation rearing (14). This could be an important confounding variable when assessing the effects of isolation on PPI, since rats are frequently manipulated during routine cage cleaning.

Therefore, the aim of the present study was to determine if minimal changes in animal manipulation during routine cage cleaning can affect the PPI deficit induced by isolation rearing.

Male Wistar rats were used. The pups remained with their mothers (7 pups per mother) until weaning (21 days) in a temperature-controlled room $\left(23 \pm 1^{\circ} \mathrm{C}\right)$ on a $12: 12$ h light:dark cycle (lights on from 6:30 to $18: 30 \mathrm{~h}$ ), with free access to food and water, and were handled three times a week (days of transfer to a clean cage). Handling consisted in grasping the rat by the tail and moving it to a clean cage (approximately 5 s), which is the typical maneuver performed by cage-changing personnel. All animal handling was performed by the same person. At weaning, the pups were allocated randomly to one of three conditions: 1$)$ grouped $(\mathrm{N}=$ 13), housed 5 per cage and handled three times a week; 2) handled-isolated ( $\mathrm{N}=15)$, housed individually and handled three times a week; 3) minimally handled-isolated ( $\mathrm{N}=$ 11 ), housed individually and receiving no handling except for cage changing once a week. Animals (grouped or isolated) were housed in $48.5 \times 25.8 \times 15.6-\mathrm{cm}$ plastic cages and could see, hear, and smell the other animals. Behavioral tests began 10 weeks after weaning and were conducted during the light phase of the daily cycle. The experiments were carried out according to the Brazilian Society of Neuroscience and Behavior guidelines for care and use of laboratory animals, and all efforts were made to minimize animal suffering.

Exploratory activity. This was tested in a novel environment using a circular $(72 \mathrm{~cm}$ in diameter) open arena surround by $49-\mathrm{cm}$ high Plexiglas walls. The floor was divided into twelve sections of the same area. The 
animals were placed in the middle of the arena and the number of floor section crossings (horizontal exploration) and the number of rearings (standing with the forelegs raised within the arena or against the walls, vertical exploration) were scored every minute for 5 min.

Prepulse inhibition of the startle reflex. Two separate stabilimeter devices were used simultaneously to record the amplitude of the acoustic startle response in the PPI test. The rats were placed in a stabilimeter which consisted of a wire-mesh cage (16.5 x $5.1 \times$ $7.6 \mathrm{~cm}$ ) and a response platform suspended on compression strings within a PVC frame $(25 \times 9 \times 9 \mathrm{~cm})$, which was attached to the response platform with 4 thumbnail-screws. The stabilimeter was located inside a ventilated plywood sound-attenuating chamber $(64 \times 60 \times 40 \mathrm{~cm})$. The floor of the wiremesh cage consisted of six stainless steel bars $3.0 \mathrm{~mm}$ in diameter spaced $1.5 \mathrm{~mm}$ apart. The startle reaction of the rats generated a pressure on the response platform and analog signals were amplified, digitized and analyzed by a software (Startle reflex, version 4.10, Med Associates Inc., St. Albans, VT, USA), which also controlled all parameters of the session (intensity of the acoustic stimulus, inter-stimulus interval, etc.). The startle amplitude was recorded within a time window of $200 \mathrm{~ms}$ after the onset of the startle stimulus. A loudspeaker located 10 $\mathrm{cm}$ behind the wire-mesh cage was used to deliver both the acoustic startle stimuli and a continuous background noise (white noise, $55 \mathrm{~dB}$ ). Calibration procedures were conducted before the experiments to ensure equivalent sensitivities of the response platforms over the test period. Animal behavior was recorded by a video camera (Everfocus, San Marino, CA, USA) located behind the stabilimeter, allowing the discrimination of all possible behaviors, with the signal being relayed to a monitor in another room via a closed circuit. A red light bulb $(6.0 \mathrm{~W})$ was located on the floor of the isolation chamber to provide illumination for the camera.

The sessions of the PPI test consisted of three parts. The first was a 5-min period of acclimatization to the startle test chamber. No acoustic startle stimuli were presented during this period. The second part consisted of ten presentations of an acoustic startle stimulus $(100-\mathrm{dB}, 50-\mathrm{ms}$ bursts of white noise having a rise-decay time of 5 $\mathrm{ms}$ ) with an inter-stimulus interval of $30 \mathrm{~s}$. The purpose was to determine the baseline startle response and to allow within-session habituation to the startle stimulus. The third part tested the level of reduction of the magnitude of the acoustic startle by prior presentation of the prepulse stimulus. It consisted of 60 trials divided into three different categories (20 trials each), i.e., pulse alone (startle stimulus, $100 \mathrm{~dB}$ of white noise, 50 $\mathrm{ms})$, prepulse alone $(80 \mathrm{~dB}$ of pure tone, $3,000 \mathrm{~Hz}$ frequency, $20 \mathrm{~ms}$ ) and prepulse + pulse (with a 30-ms interval between them), presented at random order with an interstimulus interval of $30 \mathrm{~s}$. The testing session lasted $50 \mathrm{~min}$. The startle response amplitudes obtained from this experiment were stored on a hard disk and subsequently transferred to tables in a spreadsheet program (Excel; Microsoft Corp., Montain View, CA, USA) for the off-line analysis.

Since the order of the exploratory activity and PPI tests (12), the interval between tests, and the number of times that the animals are retested $(2,6)$ may interfere with the PPI results, in the present study the two tests were performed only once. The PPI test was carried out $24 \mathrm{~h}$ after the isolation period and the exploratory activity in an open arena 3 days later.

The results of the exploratory activity are reported as means \pm SEM. Data obtained for each recorded behavioral category in the grouped, handled-isolated and minimally handled-isolated animals were analyzed by two-way analysis of variance (ANOVA), with handling and time in the arena as between-subject factors. PPI data are reported 
Figure 1. $A, B$, Exploratory activity measured in the open field. Data are reported as mean \pm SEM number of crossings (horizontal exploration, A) or rearings (vertical exploration, B). C, Percent of prepulse inhibition. $\mathrm{N}=$ 13 for grouped rats, $N=11$ for minimally handled-isolated rats, and $\mathrm{N}=15$ for handled isolated rats. ${ }^{*} \mathrm{P}<0.05$ compared to both grouped and handled-isolated groups (one-way ANOVA followed by the Newman-Keuls test). as \%PPI \{100 - [(prepulse + pulse $) \times 100] /$ pulse $\}$. Grouped, handled-isolated and minimally handled-isolated animals were compared by one-way ANOVA followed by the Newman-Keuls test. $\mathrm{P}<0.05$ was considered to be statistically significant.

There was no difference in horizontal exploratory activity (crossings) when grouped, handled-isolated and minimally handled-isolated animals were compared $\left(\mathrm{F}_{2,180}=0.925\right.$, $\mathrm{P}>0.05$, Figure 1A). However, for both groups the number of crossings was significantly higher during the first minutes in the arena, declining over the $5 \mathrm{~min}$ of recording

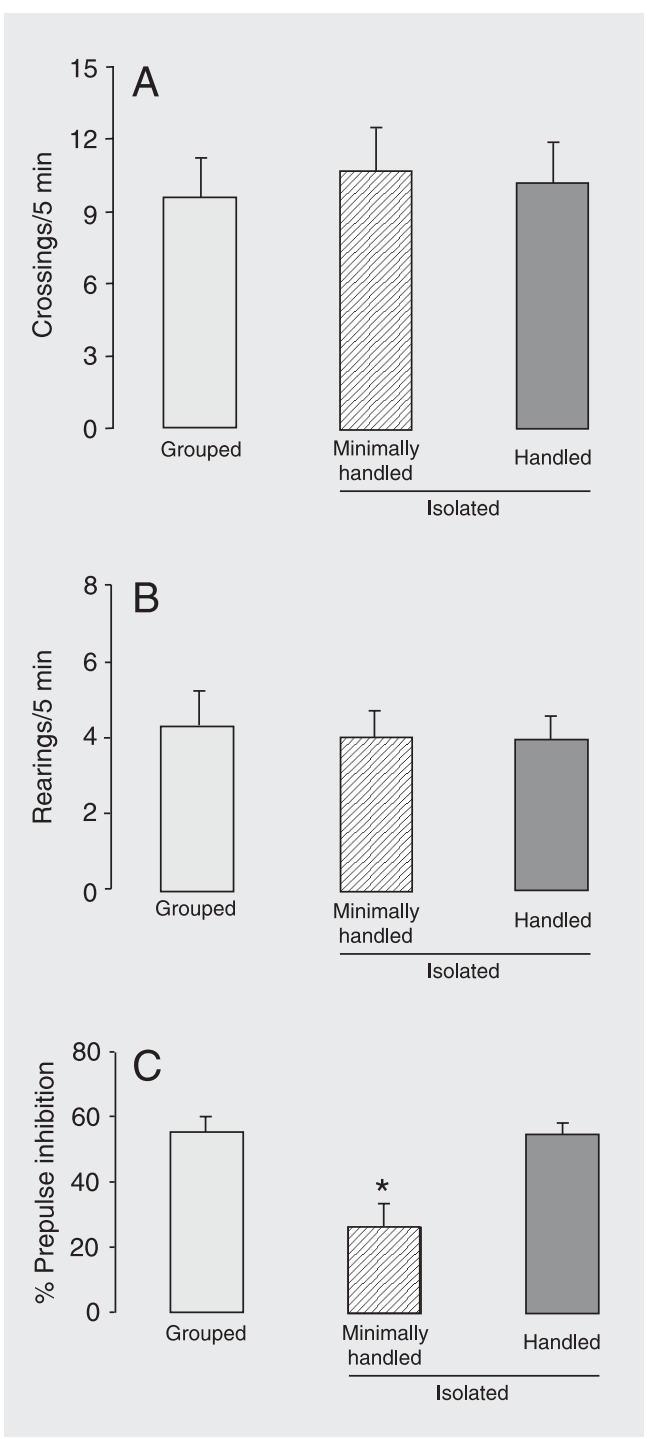

$\left(\mathrm{F}_{4,180}=55.803, \mathrm{P}<0.0001\right)$, showing the habituation of the animals to the test environment. There was no interaction between handling and time in the arena for crossings $\left(\mathrm{F}_{8,180}=0.693, \mathrm{P}>0.05\right)$. When the overall vertical exploratory activity (rearings) was compared between grouped and isolated animals, no significant difference was found $\left(\mathrm{F}_{2,180}=0.397, \mathrm{P}>0.05\right.$, Figure 1B). However, the number of rearings during the 5 min in the arena was significantly different $\left(\mathrm{F}_{4,180}=9.561, \mathrm{P}<0.0001\right)$. There was a barely significant interaction between handling and time in the arena for rearings $\left(\mathrm{F}_{8,180}\right.$ $=1.848, \mathrm{P}=0.071)$. Grouped $\left(\mathrm{F}_{4,60}=7.94\right.$, $\mathrm{P}<0.0001)$ and handled-isolated groups $\left(\mathrm{F}_{4,70}=3.64, \mathrm{P}<0.01\right)$ showed habituation while the minimally handled-isolated group $\left(\mathrm{F}_{4,50}=1.07, \mathrm{P}>0.05\right)$ showed lower but similar numbers of rearings maintained during the $5 \mathrm{~min}$ in the arena, suggesting that the animals presented no habituation to the new environment.

In the PPI test, the minimally handledisolated animals showed a significant reduction $(52 \pm 4.1 \%)$ in PPI $\left(\mathrm{F}_{9,47}=36, \mathrm{P}=\right.$ 0.0005) when compared to both grouped and handled-isolated groups (Figure 1C). No significant difference in PPI levels was found between grouped and handled-isolated animals.

Confirming previous literature results $(12,14)$, ten weeks of post-weaning social isolation induced a PPI deficit. However, this deficit was found only in animals that had received minimal handling (once a week). When the animals, even isolated, were handled three times a week, the PPI deficit was not detected. Our results agree with the reports of Krebs-Thomson et al. (14), which show that post-weaning handling of isolation-reared rats disrupts the PPI deficit. In that study the isolated animals were handled daily, held around the torso area and brought up to the handler's chest for $10 \mathrm{~s}$. Our study shows that handling the isolated animals just three times a week by 
grasping by the tail for a few seconds is enough to attenuate the PPI deficit. This suggests that the type and frequency of handling are variables that must be controlled systematically when the isolation-rearing paradigm with PPI is used as a dependent measure.

Hall et al. (15) showed that handling and testing animals in an open field three times a week after weaning did not affect the increased basal levels of DA in the nucleus accumbens induced by 8 weeks of isolation. They also showed that isolation or handling increased DA release induced by d-amphetamine whereas no interaction between these two variables was found, suggesting that rearing and handling have independent but additive effects on DA release in the nucleus accumbens. However, while isolation did not affect the 5-HT system in the nucleus accumbens, handling or interaction of isolation and handling increased it. The findings show that both isolation rearing and repeated handling/testing early during development can lead to similar, but not identical, behavioral and neurochemical changes, suggesting that these two conditions do not involve the same general mechanism. Thus, animal care during isolation rearing is very important when this methodological approach is used to study the neurochemical changes underlying the brain alterations that occur in psychiatric disorders.

Besides the reduction in PPI of the startle response, several reports suggest that rats reared in isolation are hyperactive in a novel environment such as an open field $(2,6,8,12)$. In our study, however, no change in overall exploratory activity following isolation rearing was found when the animals were tested in the open field. Because the isolated rats stayed in the same room during isolation, where they could see, smell, and hear the other animals, and where the open field test was performed, the apparatus might not have represented a novel environment to the animals. Furthermore, our findings showed that both handled-isolated and minimally handled-isolated animals presented a reduction in rearing. In agreement with this, it has been shown that handling prevents the hyperactivity induced by isolation (16). However, it has also been shown that grouped rats or handled-isolated rats are very active in an open field while non-handled isolated rats freeze in a corner of the apparatus (13). These findings demonstrate that small differences in rearing conditions can produce large differences in the rat response to the open field.

The main result of the present study is that post-weaning manipulation of rats during isolation rearing can prevent the impairments of PPI, an index of the functioning of the sensorimotor gating. To be effective, there is no need of a specialized type of handling as outlined above (14), a minimal of routine handling is sufficient to counteract the deleterious effects of isolation on the sensorimotor gating. Taken together, the present findings indicate that the combined use of the isolation rearing and prepulse inhibition may be a useful animal model for studying the sensorimotor gating deficiency characteristic of schizophrenia.

\section{Acknowledgments}

We thank Célia Aparecida da Silva and Andressa Pollo Barion for technical support. 


\section{References}

1. Kim JW \& Kirkpatrick B (1996). Social isolation in animal models of relevance to neuropsychiatric disorders. Biological Psychiatry, 40: 918-922.

2. Geyer MA, Wilkinson SL, Humby T et al. (1993). Isolation rearing of rats produces a deficit in prepulse inhibition of acoustic startle similar to that in schizophrenia. Biological Psychiatry, 34: 361-372.

3. Fulford AJ \& Marsden CA (1998). Conditioned release of 5-hydroxytryptamine in vivo in the nucleus accumbens following isolationrearing in the rat. Neuroscience, 83: 481-487.

4. Heidbreder CA, Weiss IC, Domeney AM et al. (2000). Behavioral, neurochemical and endocrinological characterization of the early social isolation syndrome. Neuroscience, 100: 749-768.

5. Swerdlow NR, Braff DL \& Geyer MA (1999). Cross-species studies of sensorimotor gating of the startle reflex. Annals of the New York Academy of Sciences, 877: 202-216.

6. Varty GB \& Higgins GA (1995). Examination of drug-induced and isolation-induced disruptions of prepulse inhibition as models to screen antipsychotic drugs. Psychopharmacology, 122: 15-26.

7. Hall FS, Huang S \& Fong G (1997). Effects of isolation-rearing on acoustic startle and prepulse inhibition in Wistar and Fawn Hooded rats. Annals of the New York Academy of Sciences, 821: 542-544.

8. Wilkinson LS, Killcross SS, Humby T et al. (1994). Social isolation in the rat produces developmentally specific deficits in prepulse inhibition of the acoustic startle response without disrupting latent inhibition. Neuropsychopharmacology, 10: 61-72.

9. Bakshi VP \& Geyer MA (1999). Ontogeny of isolation rearing-in- duced deficits in sensorimotor gating in rats. Physiology and Behavior, 67: 385-392.

10. Varty GB, Braff DL \& Geyer MA (1999). Is there a critical developmental 'window' for isolation rearing-induced changes in prepulse inhibition of the acoustic startle response? Behavioural Brain Research, 100: 177-183.

11. Weiss IC, Feldon J \& Domeney AM (1999). Isolation rearing-induced disruption of prepulse inhibition: further evidence for fragility of the response. Behavioural Pharmacology, 10: 139-149.

12. Domeney A \& Feldon J (1998). The disruption of prepulse inhibition by social isolation in the Wistar rat: how robust is the effect? Pharmacology, Biochemistry, and Behavior, 59: 883-890.

13. Holson RR, Scallet AC, Ali SF et al. (1991). "Isolation stress" revisited: Isolation-rearing effects depend on animal care methods. Physiology and Behavior, 49: 1107-1118.

14. Krebs-Thomson K, Giracello D, Solis A et al. (2001). Post-weaning handling attenuates isolation-rearing induced disruptions of prepulse inhibition in rats. Behavioural Brain Research, 120: 221-224.

15. Hall FS, Wilkinson LS, Humby T et al. (1998). Isolation rearing in rats: pre- and postsynaptic changes in striatal dopaminergic systems. Pharmacology, Biochemistry, and Behavior, 59: 859-872.

16. Gentsch C, Lichtsteiner M, Frischknecht HR et al. (1988). Isolationinduced locomotor hyperactivity and hypoalgesia in rats are prevented by handling and reversed by resocialization. Physiology and Behavior, 43: 13-16. 